\title{
The Rational Invariants of the Tame Quivers
}

\section{Claus Michael Ringel}

Fakultät für Mathematik, Universität Bielefeld, Universitätsstraße, D-4800 Bielefeld, Federal Republic of Germany

The notions of a quiver and its representations were introduced by P. Gabriel [6] in order to formalize certain matrix calculations and subspace arguments and in order to investigate algebras of finite representation type. Recall that a quiver $\Gamma$ is given by a set $\Gamma_{0}$ of "points" and a set $\Gamma_{1}$ of "arrows" such that for every arrow $\alpha \in \Gamma_{1}$, there is assigned its source $\alpha^{\prime} \in \Gamma_{0}$ and its sink $\alpha^{\prime \prime} \in \Gamma_{0}$.

Let $k$ be a field. A representation $V=\left(V_{i}, \varphi_{\alpha}\right)$ of $\Gamma$ is given by finite dimensional vector spaces $V_{i}$, for $i \in \Gamma_{0}$, and linear maps $\varphi_{\alpha}: V_{\alpha^{\prime}} \rightarrow V_{\alpha^{\prime \prime}}$, for $\alpha \in \Gamma_{1}$. (Maps will be written on the right of the argument, thus $f g$ denotes the composition of first $f$, then $g$.) If $V_{i}$ is of dimension $d_{i}$, then we call $\mathbf{d}=\left(d_{i}\right)_{i}$ the dimension type of $V$, and usually we will assume that $V_{i}=k^{d_{2}}$. If we fix $a^{\prime}$, the set of all representations of $\Gamma$ of dimension type $\mathbf{d}$ forms the affine space

$$
\mathbb{A}[\Gamma, \mathbf{d}]=\prod_{\alpha \in \Gamma_{1}} \operatorname{Hom}\left(k^{d_{\alpha^{\prime}}}, k^{d_{\alpha^{\prime \prime}}}\right) .
$$

The ring of regular functions on $\mathbb{A}[\Gamma, \mathbf{d}]$ is

$$
k[\Gamma, \mathbf{d}]=k\left[X_{\alpha s t} \mid \alpha \in \Gamma_{1}, 1 \leqq s \leqq d_{x^{\prime}}, 1 \leqq t \leqq d_{\alpha^{\prime \prime}}\right],
$$

the corresponding ring of rational functions will be denoted by $k(\Gamma, \mathbf{d})$. There is a canonical group action on $\mathbb{A}[\Gamma, \mathbf{d}]$, and correspondingly on $k[\Gamma, \mathbf{d}]$, and $k(\Gamma, \mathbf{d})$, by the algebraic group

$$
G(\mathbf{d})=\prod_{i \in \Gamma_{0}} \mathrm{Gl}\left(d_{i}, k\right)
$$

such that the orbits of $\mathbb{A}[\Gamma, \mathbf{d}]$ under $G(\mathbf{d})$ correspond bijectively to the isomorphism classes of representations of $\Gamma$ of dimension type $\mathbf{d}$. Assume $\Gamma$ has no oriented cycle. In this case, the zero element $(0)_{\alpha s t}$ of $\mathbb{A}[\Gamma, \mathbf{d}]$ belongs to the closure of any orbit under $G(\mathbf{d})$, thus it is clear that there do not exist non-trivial polynomial invariants. In this paper, we are concerned with the determination of rational invariants. There also will be no non-trivial rational invariants in case $\Gamma$ if of finite representation type and we recall that Gabriel [6] has shown that this 
happens if and only if $\Gamma$ is a disjoint union of Dynkin diagrams $\mathbf{A}_{n}, \mathbf{D}_{n}, \mathbf{E}_{6}, \mathbf{E}_{7}$, $\mathbf{E}_{8}$.

Our aim, in this paper, is to consider the so-called tame cases $\tilde{\mathbf{A}}_{n}, \tilde{\mathbf{D}}_{n}, \tilde{\mathbf{E}}_{6}, \tilde{\mathbf{E}}_{7}$, $\tilde{\mathbf{E}}_{8}$ of extended Dynkin diagrams. In these cases, it is rather easy to see that the field $I(I, \mathbf{d})$ of rational invariants is a purely transcendental extension of $k$, and we will give an explicit formula for generators of $I(\Gamma, \mathbf{d})$. We will use the known classification of the indecomposable representations of the tame quivers due to Donovan-Freislich [5] and Nazarova [13], several special cases being known for some time: the case $\tilde{\mathbf{A}}_{1}$, the socalled matrix pencils, was solved by Kronecker [11] in 1890, the four subspace situation $\tilde{\mathbf{D}}_{4}$ was treated by Nazarova [12] and Gelfand-Ponomarev [9]. Note that a rational invariant is of the form $f / g$ with $f$, $g$ semi-invariants with same weight. In case $\Gamma$ is tame, we will construct semiinvariants $f_{0}, \ldots, f_{p}$ for a fixed weight $\partial$ such that the elements

$$
\frac{f_{1}}{f_{0}}, \ldots, \frac{f_{p}}{f_{0}}
$$

form a transcendence basis of $I(I, \mathbf{d})$. It is of interest that this $\partial$ is just the (normalized) defect, a concept which played a fundamental role in one of the proofs of the classification theorem for the indecomposable representations $([5,4])$. Also, the regular representations in the sense of [2] turn out to be precisely those elements of $\mathbb{A}[\Gamma, \mathbf{d}]$ in which the rational map $\left(f_{0}: \ldots: f_{p}\right)$ : $\mathbb{A}[\Gamma, \mathbf{d}] \rightarrow \mathbb{P}_{p}$ is regular.

We should mention that one particular rational invariant, namely for the four subspace quiver (of type $\tilde{\mathbf{D}}_{4}$ ) and the dimension type $\mathbf{d}=(1,1,1,1,2$ ), is well-known to everyone: the cross ratio of the corresponding points on the projective line $\mathbb{P}_{1}$. Our investigation gives, in particular, a complete set of rational invariants for the position of four arbitrary subspaces in an arbitrary projective space. We believe that this should be of interest to classical geometers. In the same way, we also obtain complete sets of rational invariants for the other subspace situations given by the diagrams $\tilde{\mathbf{E}}_{6}, \tilde{\mathbf{E}}_{7}, \tilde{\mathbf{E}}_{8}$.

The investigations of this paper have their origin in the joint work with $\mathrm{V}$. Dlab on the representation theory of tame species, in particular the determination of conditions which force a representation to be homogeneous, see the tables of [4]. In fact, it turns out that in this way certain semi-invariants had been calculated. The problem of computing the invariants and the orbit structure in the tame case was suggested by V. Kac. The author is indebted to Dlab, $\mathrm{Kac}$, and also C. Procesi for many helpful discussions concerning these questions. He also thanks the referee for pointing out the short proof of Lemma 2.5 given here.

\section{Review on the Representation Theory of Quivers}

We always will assume that $\Gamma$ is a quiver with $n$ points and without oriented cycles. In this case, the indecomposable projective representations are finite dimensional. 
Given a representation $V=\left(V_{i}, \varphi_{x}\right)$ of $\Gamma$, we have introduced its dimension type $\mathbf{d}=\left(d_{i}\right)_{i}$, where $d_{i}=\operatorname{dim} V_{i}$, and write $\mathbf{d}=\operatorname{dim} V$. This is an element of $\mathbb{N}^{n}$, and we will consider it sometimes as an element of the rational vector space $\mathfrak{D}$ $=\mathbb{Q}^{n}$. We denote by $\mathfrak{D}^{*}$ the dual space.

On $\mathfrak{D}$, there is given a quadratic form $q$ as follows:

$$
q(\mathbf{x})=\sum_{i \in \Gamma_{0}} x_{i}^{2}-\sum_{\alpha \in \Gamma_{t}} x_{\alpha^{\prime}} x_{\alpha^{\prime \prime}}
$$

This quadratic form is positiv definite if and only if $\Gamma$ is a disjoint union of Dynkin diagrams $\mathbf{A}_{n}, \mathbf{D}_{n}, \mathbf{E}_{6}, \mathbf{E}_{7}, \mathbf{E}_{8}$, and it is semi-definite for $\tilde{\mathbf{A}}_{n}, \tilde{\mathbf{D}}_{n}, \tilde{\mathbf{E}}_{6}, \tilde{\mathbf{E}}_{7}$, $\tilde{\mathbf{E}}_{8}$. In these cases, there is a one-dimensional subspace of $\mathfrak{D}$ on which $q$ vanishes, and this subspace contains vectors with positive integral components, the minimal one will be denoted by $\mathbf{h}$.

Our main working tool will be Coxeter functors as introduced by Bernstein, Gelfand and Ponomarev [2]. In fact, it will be more convenient to use the twisted analogues considered by Auslander, Platzek and Reiten, thus let $C^{+}$ $=D \operatorname{Tr}$, and $C^{-}=\operatorname{Tr} D$, see [1]. These functors coincide with the original Coxeter functors of [2] up to a categorial equivalence (see [1] and also [8]). Note that for an indecomposable representation $V$, either $V$ is projective and then $C^{+} V$ $=0$, or else $C^{+} V$ is indecomposable again, $V \approx C^{-} C^{+} V$, and $\operatorname{dim} C^{+} V$ $=c \operatorname{dim} V$, where $c$ is a linear transformation on $\mathcal{D}$, called the Coxeter transformation. The functor $C^{+}$can be used in order to calculate the dimension of Extgroups. (Since we deal with a hereditary category, we just write Ext for $\operatorname{Ext}^{1}$.) Given two representations $V, W$, the vectorspaces $\operatorname{Ext}(V, W)^{*}$ and $\operatorname{Hom}\left(W, C^{+} V\right)$ are naturally isomorphic [1], in particular, we have

$$
\operatorname{dim} \operatorname{Ext}(V, W)=\operatorname{dim} \operatorname{Hom}\left(W, C^{+} V\right) .
$$

The direct sums of representations of the form $C^{-n} P$, with $P$ indecomposable projective and $n \in \mathbb{N}$, are called preprojective. Similarly, the direct sums of representations of the form $C^{+n} I$, with $I$ indecomposable injective, and $n \in \mathbb{N}$, are called preinjective.

In case $\Gamma$ is a disjoint union of Dynkin diagrams, and only in this case, all representations are both preprojective and preinjective; this is just the case when $\Gamma$ is of finite representation type. If $\Gamma$ is not of finite representation type, there will be indecomposable representations which are neither preprojective, nor preinjective; they are called regular. More generally, an arbitrary representation is called regular [2] if it has no non-zero preprojective or preinjective direct summand. We denote by $\mathfrak{D}_{r}$ the subset of $\mathfrak{D}$ consisting of the dimension types $\operatorname{dim} V$, with $V$ regular.

Now assume that $\Gamma$ is tame (and connected), thus the underlying graph is of the form $\tilde{\mathbf{A}}_{n}, \tilde{\mathbf{D}}_{n}, \tilde{\mathbf{E}}_{6}, \tilde{\mathbf{E}}_{7}$, or $\tilde{\mathbf{E}}_{8}$. In this case, there exists an element $\partial \in \mathcal{D}^{*}$, the defect, such that for an indecomposable representation $X$, we have $\partial(\operatorname{dim} X)<0$, $=0$, or $>0$, if and only if, $X$ is preprojective, regular, or preinjective, respectively. We will always choose $\partial$ "normalized" (that is, $\partial(\mathbf{x}) \in \mathbb{Z}$, for $\mathbf{x}$ integral, and there exists $\mathbf{x}$ integral with $\partial(\mathbf{x})=1)$, and we will denote $\partial_{i}=\partial\left(\mathbf{b}_{i}\right)$ where $\mathbf{b}_{i}$ $=(0, \ldots, 0,1,0, \ldots, 0)$ is the $i$-th canonical basis vector. 
Since regular modules are of defect 0 , we see that $\mathfrak{D}_{r}$ is contained in the hyperplane of $\mathfrak{D}$ defined by the equation $\partial(\mathbf{x})=0$. However, not every positive integral vector in this hyperplane belongs to $\mathcal{D}_{r}$, for example in $\tilde{\mathbf{A}}_{u, v}$, with $u \geqq v \geqq 2$, dimension types $\mathbf{x}$ given by

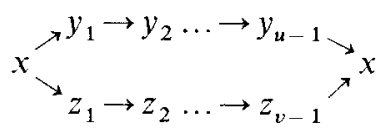

all satisfy $\partial(\mathbf{x})=0$, however there exists a regular representation $V$ of dimension type $\mathbf{x}$ only in case $x \leqq \min _{1 \leqq i \leqq u} y_{i}+\min _{1 \leqq i \leqq v} z_{i}$. In the tame case, a complete classification of the regular representations is known. Namely, the regular representations form an abelian category $\mathscr{R}$, thus we may speak of regular composition series, simple regular objects, etc referring to composition series, simple objects, etc inside the category $\mathscr{R}$. Now $\mathscr{R}$ is a serial category: any indecomposable regular representation has a unique regular composition series, thus it is uniquely determined by its regular socle and its regular length.

A simple regular module $E$ will be called homogeneous, provided $\operatorname{dim} E$ is a multiple of $\mathbf{h}$, or, equivalently, provided $\operatorname{dim} E$ is fixed under the Coxeter transformation $c$. In fact, for any simple regular module $E$, the orbit of $\operatorname{dim} E$ under $c$ is always finite, and there are at most three orbits which contain more than one element. Let $E_{i}, i \in I$, be the non-homogeneous simple regular representations, and let $\mathbf{e}_{i}=\operatorname{dim} E_{i}$. Note that $E_{i}$ completely is determined by $\mathbf{e}_{i}$. We call a subset $J \subseteq I$ linear (in contrast to circular), provided the set $\left\{\mathbf{e}_{j} \mid j \in J\right\}$ does not contain a complete $c$-orbit. Then it follows that the elements $\mathbf{h}, \mathbf{e}_{j}, j \in J$, are linearly independent in $\mathfrak{D}$.

Recall that $\mathfrak{D}_{r}$ denotes the dimension types of regular representations. It is clear that in the tame case, any $\mathbf{d} \in \mathcal{D}_{r}$ is of the form $\mathbf{d}=p \mathbf{h}+\sum_{i \in I} p_{i} \mathbf{e}_{i}$, with $p, p_{i} \in \mathbb{N}$. Since the sum of all elements $\mathbf{e}_{i}$ in one $c$-orbit is just $\mathbf{h}$, we can assume that $\mathbf{d}=p \mathbf{h}+\sum_{i \in I} p_{i} \mathbf{e}_{i}$, satisfies the following conditions: all $p, p_{i} \in \mathbb{N}$, and for every $c$-orbit $I^{\prime}$, there exists $\mathbf{e}_{i} \in I^{\prime}$, such that $p_{i}=0$. In this case, we call this the canonical decomposition of $\mathbf{d} \in \mathfrak{D}_{r}$. Note that for $\mathbf{d} \in \mathfrak{D}_{r}$, the canonical decomposition is unique.

Finally, we note that we get a decomposition of $\mathscr{R}$ as the direct sum of categories $\mathscr{R}_{t}, t \in \mathbb{T}$, where as index set $\mathbb{T}$ we may take the set obtained from the set of irreducible monic polynomials over $k$ by adjoining one additional element $\infty$. (Thus, if $k$ is algebraically closed, $\mathbb{T}=\mathbb{P}_{1}(k)$.) In all but at most three categories $\mathscr{R}_{t}$, say except perhaps for $t \in\{0,1, \infty\}$, there is just one simple object, and this then has to be homogeneous. In any one of the exceptional categories $\mathscr{R}_{t}$, one simple regular representation will be of interest to us, and we will denote it by $E_{t}$ (see Sect. 5). In particular, we will assume that the index set $I$ of the nonhomogeneous simple regular representations contains these values $t$. We denote by $n_{t}$ the number of simple regular representations in $\mathscr{R}_{t}$.

We will also have to consider the special quiver $\Delta=\bullet \supset$ (which has an oriented cycle). Note that the representations of $\Delta$ of dimension type $p$ are just 
the $p \times p$ matrices, and isomorphism of representations means just equivalence of matrices. Besides this, the special case of the quiver $\Sigma=V_{;}$of type $\tilde{\mathbf{A}}_{1}$ (which was studied by Kronecker) will be of importance.

\section{The Open Sheet}

If an algebraic group $G$ acts on the affine space $\mathbb{A}^{m}$, the stabilizer dimension is semi-continuous on $\mathbb{A}^{m}$; in particular, the set of points $V \in \mathbb{A}^{m}$ with minimal stabilizer dimension is an open (and therefore also dense) set, called the open sheet of $\mathbb{A}^{m}$ under $G$. (In general, the sheets are the irreducible components of the various sets $\left\{V \in \mathbb{A}^{m} \mid\right.$ stabilizer dimension of $V$ is $\left.i\right\}$, with $i \in \mathbb{N}$.).

In our case $\mathbb{A}^{m}=\mathbb{A}[\Gamma, \mathbf{d}], G=G(\mathbf{d})$, the stabilizer of any $V=\left(\varphi_{\alpha s t}\right)$ is precisely the automorphism group of the reprentation $V$, thus its dimension is equal to the dimension of the endomorphism ring End $(V)$ of $V$. In determining conditions for representations to belong to the open sheet, the following lemma is useful.

Lemma 2.1. Let $0 \rightarrow U \stackrel{\mu}{\rightarrow} V \stackrel{\pi}{\rightarrow} W \rightarrow 0$ be a non-split exact sequence. Then $\operatorname{dim} \operatorname{End}(V)<\operatorname{dim} \operatorname{End}(U \oplus W)$.

Proof. We may suppose that $\mu$ is an inclusion. Let

$$
\begin{aligned}
& H_{0}=\{\alpha \in \operatorname{End}(V) \mid V \alpha \subseteq U, U \alpha=0\}, \\
& H_{1}=\{\alpha \in \operatorname{End}(V) \mid U \alpha \subseteq U\} .
\end{aligned}
$$

Then $\operatorname{Hom}(W, U) \approx H_{0}$ under $\beta \mapsto \pi \beta \mu$, and $\operatorname{End}(V) / H_{1} \hookrightarrow \operatorname{Hom}(U, W)$ under $\alpha \mapsto \mu \alpha \pi$. Also $H_{1} / H_{0}$ embeds into End $(U) \times \operatorname{End}(W)$ under $\alpha \mapsto\left(\alpha_{U}, \alpha_{W}\right)$, where for $\alpha \in H_{1}$, the endomorphisms $\alpha_{U}$ of $U$ and $\alpha_{W}$ of $W$ are defined by $\mu \alpha=\alpha_{U} \mu, \alpha \pi$ $=\pi \alpha_{W}$. Altogether, this shows that $\operatorname{dim} \operatorname{End}(V) \leqq \operatorname{dim} \operatorname{End}(U \oplus W)$. Now assume we have equality, then the constructed inclusion both have to be surjective. In particular, the last one: thus, there is $\alpha \in H_{1}$ with $\alpha_{U}=1$ and $\alpha_{W}=0$. Clearly, this gives a splitting of $\mu$, since $\alpha_{W}=0$ means that $\alpha$ maps into $U$, and $\alpha_{U}=1$.

Note that the lemma also could be proved using some elementary arguments from the theory of operations of algebraic groups.

Corollary 2.2. Let $V$ belong to the open sheet of $\mathbb{A}[\Gamma, \mathbf{d}]$ under $G(\mathbf{d})$. Then, for any decomposition $V=V^{\prime} \oplus V^{\prime \prime}$, we have $\operatorname{Ext}\left(V^{\prime}, V^{\prime \prime}\right)=0$.

Proof. Any non-trivial exact sequence

$$
0 \rightarrow V^{\prime \prime} \rightarrow W \rightarrow V^{\prime} \rightarrow 0
$$

would produce a representation $W$ belonging to $\mathbb{A}[\Gamma, \mathbf{d}]$ but with smaller endomorphism ring dimension.

Recall from [14] that there exists a (non-symmetric) bilinear form $b$ on $\mathbb{Q}^{n}$ which induces the usual quadratic form $q$ and such that for any two representations $V, W$, we have

$$
b(\operatorname{dim} V, \operatorname{dim} W)=\operatorname{dim} \operatorname{Hom}(V, W)-\operatorname{dim} \operatorname{Ext}(V, W) .
$$


The usual quadratic form satisfies

$$
q(\mathbf{d})=\operatorname{dim} G(\mathbf{d})-\operatorname{dim} \mathbb{A}[\Gamma, \mathbf{d}]
$$

for $\mathbf{d} \in \mathbb{N}^{n}$. Thus, if $V$ is a representation of $\Gamma$ with $\operatorname{dim} V=d$, and with orbit $\mathcal{O}(V)$ in $\mathbb{A}[\Gamma, \mathbf{d}]$, then

thus

$$
\operatorname{dim} G(\mathbf{d})=\operatorname{dim} O(V)+\operatorname{dim} \operatorname{End}(V),
$$

$$
\begin{aligned}
\operatorname{dim} \operatorname{Ext}(V, V) & =\operatorname{dim} \operatorname{End}(V)-q(\mathbf{d}) \\
& =\operatorname{dim} \operatorname{End}(V)-\operatorname{dim} G(\mathbf{d})+\operatorname{dim} \mathbb{A}[\Gamma, \mathbf{d}] \\
& =\operatorname{dim} \mathbb{A}[\Gamma, \mathbf{d}]-\operatorname{dim} \mathcal{O}(V) .
\end{aligned}
$$

This proves the following lemma which seems to be due to M. Artin and D. Voigt [7].

Lemma 2.3. Let $V$ be a representation with $\operatorname{dim} V=\mathbf{d}$. Then the codimension of the orbit $\mathcal{O}(V)$ of $V$ in $\mathbb{A}[\Gamma, \mathbf{d}]$ is equal to $\operatorname{dim} \operatorname{Ext}(V, V)$.

In particular, we have the following

Corollary 2.4. Let $V$ be a representation with $\operatorname{dim} V=\mathbf{d}$. Then the orbit $O(V)$ is dense in $\mathbb{A}[\Gamma, \mathbf{d}]$ if and only if $\operatorname{Ext}(V, V)=0$.

Lemma 2.5. Let $U, V$ be representations in $\mathbb{A}[\Gamma, \mathbf{d}]$. Assume $U$ has no non-zero preprojective direct summand, and assume that for any direct decomposition $V$ $=V^{\prime} \oplus V^{\prime \prime}$, we have $\operatorname{Ext}\left(V^{\prime}, V^{\prime \prime}\right)=0$. Then $V$ has no non-zero preprojective direct summand.

Proof. Assume $V=P \oplus M$ for some indecomposable preprojective module $P$, say with $\operatorname{dim} P=\mathbf{p}$. Since $U$ has no non-zero preprojective direct summand, $\operatorname{Hom}(U, P)=0$, thus

$$
b(\mathbf{d}, \mathbf{p})=\operatorname{dim} \operatorname{Hom}(U, P)-\operatorname{dim} \operatorname{Ext}(U, P)=-\operatorname{dim} \operatorname{Ext}(U, P) \leqq 0 .
$$

By assumption, $\operatorname{Ext}(M, P)=0$. Together with $\operatorname{Ext}(P, P)=0$, this implies that $\operatorname{Ext}(V, P)=0$. Thus, we also calculate

$$
b(\mathbf{d}, \mathbf{p})=\operatorname{dim} \operatorname{Hom}(V, P)-\operatorname{dim} \operatorname{Ext}(V, P)=\operatorname{dim} \operatorname{Hom}(V, P) \geqq 1,
$$

a contradiction.

Of course, we also have the dual assertion:

Lemma 2.5*. Let $U, V$ be representations in $\mathbb{A}[\Gamma, \mathbf{d}]$. Assume $U$ has no non-zero preinjective direct summand, and assume that for any direct decomposition $V$ $=V^{\prime} \oplus V^{\prime \prime}$, we have $\operatorname{Ext}\left(V^{\prime}, V^{\prime \prime}\right)=0$. Then $V$ has no non-zero preinjective direct summand.

Theorem 2.6. Let $\Gamma$ be a quiver, and assume there exists a regular representation of $\Gamma$ of dimension type $\mathbf{d}$. Then all representations in the open sheet of $\mathbb{A}[\Gamma, \mathbf{d}]$ are regular.

Proof. This is an immediate consequence of $2.5,2.5^{*}$, and 2.2 . 


\section{The Tame Case}

We assume now that $\Gamma$ is tame.

Lemma 3.1. Let $V=R \oplus U$ be representations of $\Gamma$, with $R$ regular, and $\operatorname{Ext}(R, U)$ $=0=\operatorname{Ext}(U, R)$. Let $\operatorname{dim} R=p \mathbf{h}+\sum_{i \in I} p_{i} \mathbf{e}_{i}$ be the canonical decomposition. Then either $p=0$ or else $U$ is also regular.

Proof. Assume $U$ has an indecomposable preprojective direct summand, say of the form $C^{-i} P$, for some indecomposable projective module $P$. Note that the dimension type of $C^{+(i+1)} R$ is $p \mathbf{h}+\sum_{i \in I} p_{\sigma(i)} \mathbf{e}_{i}$ for some permutation $\sigma$ of $I$. Now
if $p>0$, then

and therefore

$$
0 \neq \operatorname{Hom}\left(P, C^{+(i+1)} R\right) \approx \operatorname{Hom}\left(C^{-i} P, C^{+} R\right),
$$

$$
0 \neq \operatorname{dim} \operatorname{Hom}\left(U, C^{+} R\right)=\operatorname{dim} \operatorname{Ext}(R, U),
$$

contrary to our assumption. The dual argument shows that for $p>0$, the representation $U$ cannot have an indecomposable preinjective direct summand.

Recall that we have denoted by $\mathcal{D}_{r}$ the set of dimension types of regular representations.

Theorem 3.2. Let $\Gamma$ be a tame quiver, and $\mathbf{d} \notin \mathfrak{D}_{r}$. Then there is an open orbit in A $[\Gamma, \mathbf{d}]$.

Proof. Let $V$ be a representation of $\Gamma$ belonging to the open sheet of $A[C, \mathbf{d}]$. Let $V=P \oplus Q \oplus R$ with $P$ preprojective, $Q$ preinjective and $R$ regular. Since $\mathbf{d} \notin \mathfrak{D}_{r}$, we know that $P \oplus Q \neq 0$. By $2.2, \operatorname{Ext}(P \oplus Q, R)=0=\operatorname{Ext}(R, P \oplus Q)$. Thus, if we use the canonical decomposition $\operatorname{dim} R=p \mathbf{h}+\sum_{i \in I} p_{i} \mathbf{e}_{i}$, then $p=0$ according to 3.1. As a consequence, $R$ cannot have a non-zero homogeneous direct summand. But there is only a finite number of possible representations of $\Gamma$ of dimension type $\mathbf{d}$ without non-zero homogeneous direct summand. Thus, the open sheet is the union of a finite number of orbits, and therefore one of these orbits has to be open (and, in fact, is the whole open sheet).

Remark. If $\Gamma$ is wild, the assertion is no longer true. Consider for example the quiver

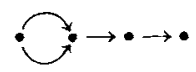

and the dimension type $\mathbf{d}=(1,1,2,1)$. It is clear that $\mathbf{d} \notin \mathfrak{D}_{r}$, since $c^{2} \mathbf{d}=(1,1,0$, -1). On the other hand, the open sheet of $\mathbb{A}[\Gamma, \mathbf{d}]$ consists of a one-parameter family (of decomposable modules).

Next, we consider $\mathbf{d} \in \mathfrak{D}_{r}$. We want to give a complete description of the representations in the open sheet of $\mathbb{A}[\Gamma, \mathbf{d}]$.

Let $I$ be the index set of the simple regular representations, and $J$ a linear subset of $I$ (that is $\left\{\mathbf{e}_{j} \mid j \in J\right\}$ does not contain a complete c-orbit). We make $J$ into a quiver by attaching an arrow $i \rightarrow j$ in case $\mathbf{e}_{j}=c\left(\mathbf{e}_{i}\right)$. Since we assume that $J$ is linear, this quiver is a disjoint union of various $A_{i}$. Let $\mathscr{R}(J)$ be the full subcategory of regular representations with regular composition factors of the form $E_{j}, j \in J$. Clearly $\mathscr{h}(J)$ is an abelian and extension closed subcategory, and is 
equivalent to the category of representations of the quiver $J$. Note that an object $V$ of $\mathscr{R}(J)$ of dimension type $\sum_{j \in J} p_{j} \mathbf{e}_{j}$ corresponds to a representation $\bar{V}$ of $J$ of
dimension type $\left(p_{j}\right)_{j \in J}$.

Let us consider for a moment representations of $J$. If $\mathbf{p}=\left(p_{j}\right)_{j \in J}$ is a dimension type, we define a representation $\vec{R}(\mathbf{p})$ inductively as follows: We call $\left\{j \in J \mid p_{j}>0\right\}$ the support of $\mathbf{p}$ (or also of any representation of dimension type $\mathbf{p}$ ). Assume the support $J^{\prime}$ of $\mathbf{p}$ has precisely $s$ connected components. Let $\bar{R}\left(J^{\prime}\right)$ be the (unique) representation of $J$ with support $J^{\prime}$ which is the direct sum of $s$ indecomposable representations, and let $\bar{R}(\mathbf{p})=\bar{R}\left(J^{\prime}\right) \oplus \bar{R}\left(\mathbf{p}^{\prime}\right)$, where $p_{i}^{\prime}=\max \left(p_{i}-1,0\right)$. The indecomposable summands of $\bar{R}\left(J^{\prime}\right)$ (and isomorphic ones) will be called the large summands of $\bar{R}(\mathbf{p})$.

Lemma 3.3. The representation $\bar{R}=\bar{R}(\mathbf{p})$ of $J$ satisfies $\operatorname{Ext}(\bar{R}, \bar{R})=0$, thus it belongs to the open orbit of $\mathbb{A}[J, \mathrm{p}]$.

Proof. We may assume that $J$ is the support of $\bar{R}(\mathbf{p})$. Then $\bar{R}(J)$ is both projective and injective in the category of representations of $J$. Thus $\operatorname{Ext}(\bar{R}(J)$. $\bar{R}(\mathbf{p}))=0=\operatorname{Ext}(\bar{R}(\mathbf{p}), \bar{R}(J))$. By induction, it follows that $\operatorname{Ext}(\bar{R}, \bar{R})=0$.

The representation in $\mathscr{R}(J)$ corresponding to $\bar{R}(\mathbf{p})$ will be denoted by $R(\mathbf{p})$, thus again we have $\operatorname{Ext}(R(\mathbf{p}), R(\mathbf{p}))=0$. Thus we have shown the first part of the following result:

Lemma 3.4. Let $\mathbf{d}=\sum_{i \in I} p_{i} \mathbf{e}_{i}$ be a dimension type with linear support. Then there exists a representation $R=R$ (d) of $\Gamma$ with $\operatorname{dim} R=\mathrm{d}$ and $\operatorname{Ext}(R, R)=0$, and any $S$ of dimension type d such that $S=S^{\prime} \oplus S^{\prime \prime}$ implies $\operatorname{Ext}\left(S^{\prime}, S^{\prime \prime}\right)=0$ is isomorphic to $R$.

Proof. Assume $S$ is of dimension $\mathbf{d}$ and satisfies the extension condition. By 2.5 , $2.5^{*}$ we know that $S$ is regular. However, any indecomposable representation $S^{\prime}$ in $\mathscr{R}(J)$ for $J$ a linear set satisfies $\operatorname{Ext}\left(S^{\prime}, S^{\prime}\right)=0$, thus it follows that $\operatorname{Ext}(S, S)=0$, and therefore the isomorphism $S \approx R$ follows from 2.4 .

The indecomposable summands of $R$ which correspond to large summands of $\bar{R}$, again will be called large. Recall that any regular representation $V$ of $\Gamma$ can be decomposed $V=\underset{t \in \mathbb{T}}{\bigoplus} V_{t}$ with $V_{t} \in \mathscr{R}_{t}$.

Theorem 3.5. Let $\Gamma$ be a tame quiver. Let $\mathbf{d} \in D_{r}$, say with canonical decomposition $\mathbf{d}=p \mathbf{h}+\sum_{i \in I} p_{i} \mathbf{e}_{i}$. Let $R=R\left(\sum_{i \in I} p_{i} \mathbf{e}_{i}\right)$. Then, for a representation $V$ of $\Gamma$ with $\operatorname{dim} V$ $=\mathbf{d}$, the following conditions are equivalent:

(i) $V$ belongs to the open sheet.

(ii) If $V=V^{\prime} \oplus V^{\prime \prime}$, then $\operatorname{Ext}\left(V^{\prime}, V^{\prime \prime}\right)=0$.

(iii) $V$ is regular, and the decomposition $V=\bigoplus_{t \in \mathbb{T}} V_{t}$ has the following properties: either $V_{t}$ is obtained from $R_{t}$ by replacing a large summand of $R_{t}$ by an indecomposable summand, or $V_{t}=W_{t} \oplus R_{t}$ where $W_{t}$ is indecomposable of dimension type a multiple of $\mathbf{h}$, and neither its regular socle nor its regular top appears as a regular composition factor of $R_{t}$.

Note. In condition (iii), assume $R_{\mathrm{t}}=R_{t}^{\prime} \oplus R_{t}^{\prime \prime}$ with $R_{t}^{\prime}$ a large summand, and $V_{t}$ $=V_{t}^{\prime} \oplus R_{t}^{\prime \prime}$ with $V_{t}^{\prime}$ indecomposable. Then, since $\operatorname{dim} V_{t}-\operatorname{dim} R_{t}$ is a multiple of $\mathbf{h}$, 
it is clear that $R_{t}^{\prime}$ embeds into $V_{t}^{\prime}$ with quotient being an indecomposable representation in $\mathscr{R}_{t}$ of dimension type a multiple of $\mathbf{h}$.

Proof. (i) $\Rightarrow$ (ii) is always true, according to 2.2 . (ii) $\Rightarrow$ (iii): We know that $V$ is regular from 2.5 and $2.5^{*}$. Clearly, we now may suppose that $V=V_{t}$ for some $t \in \mathbb{T}$ with $t \in\{0,1, \infty\}$ and $R=R_{t} \neq 0$, since for any two representations of dimension type a multiple of $\mathbf{h}$ in the same $\mathscr{R}_{t}$, there are non-trivial homomorphisms, thus non-trivial extensions.

Let $n_{t}$ be the number of simple regular representations in $\mathscr{R}_{t}$. Let $V$ $=V^{1} \oplus \ldots \oplus V^{r}$ be a direct sum decomposition with $V^{i}$ indecomposable. If all $V^{i}$ have regular length $<n_{t}$, then it is rather easy to see that we must have $V \approx R$. Thus, assume the regular length of $V^{1}$ is $\geqq n_{t}$, and let $S$ be its regular socle, and $T$ its regular top, both being simple regular. We claim that neither $C^{+} S$ nor $C^{-} T$ can occur as regular composition factor of any $V^{i}$ with $i \geqq 2$. For, assume $C^{+} S$ appears as regular composition factor of some $V^{i}$, with $i \geqq 2$, say $V^{i}$ has regular submodules $U \subset U^{\prime} \subseteq V^{i}$ with $U^{\prime} / U \approx C^{+} S$. If we choose $U$ as large as possible, then $V^{i} / U$ has regular length $\leqq n_{t}$ and therefore can be embedded into $C^{+}\left(V^{1}\right)$ (which also has $C^{+} S$ as regular socle). Thus

$$
0 \neq \operatorname{dim} \operatorname{Hom}\left(V^{i}, C^{+}\left(V^{1}\right)\right)=\operatorname{dim} \operatorname{Ext}\left(V^{1}, V^{i}\right)
$$

gives a contradiction. Similarly, if $C^{-} T \approx U / U^{\prime}$ for some regular submodules $U^{\prime} \subset U \subseteq V^{i}$ for some $i \geqq 2$, then choosing $U$ minimal, and applying $C^{+}$, we see that there exists an epimorphism $V^{1} \rightarrow C^{+} U$, thus

$$
0 \neq \operatorname{dim} \operatorname{Hom}\left(V^{1}, C^{+}\left(V^{i}\right)\right)=\operatorname{dim} \operatorname{Ext}\left(V^{i}, V^{1}\right),
$$

a contradiction.

As a consequence, we see that all $V^{i}$, with $i \geqq 2$ have regular composition length $\leqq n_{1}-1$. Also, if the regular length of $V^{1}$ is a multiple of $n_{t}$, then $C^{+} S$ $=T$, thus we get just the condition that neither $S$ nor $T$ can occur as regular composition factor of $V^{2} \oplus \ldots \oplus V^{r}$. Of course, since $V^{2} \oplus \ldots \oplus V^{r}$ has linear support, and $\operatorname{dim} V^{2} \oplus \ldots \oplus V^{r}=\operatorname{dim} R$, we conclude from 3.4 that $V^{2} \oplus \ldots \oplus V^{r} \approx R$. Finally, assume $\operatorname{dim} V^{1}=p \mathbf{h}+\sum_{j \in J} \mathbf{e}_{j}$ for some linear set $J$, and let $W$ be the regular submodule of $V^{1}$ of dimension type $\sum_{j \in J} \mathbf{e}_{j}$. Now also $W$ has regular socle $S$ and regular top $T$, and does not contain $C^{+} S$ or $C^{-} T$ as regular composition factor. It is clear that $W \oplus V^{2} \oplus \ldots \oplus V^{r}$ is of $\operatorname{dimension}$ type $\operatorname{dim} R$, and since it has linear support, it follows again from 3.4 that $R \approx W \oplus V^{2} \oplus \ldots \oplus V^{r}$. Also, since $C^{+} S$ and $C^{-} T$ do not occur as composition factors, we see that the support of $W$ is a connected component of the support of $R$, thus $W$ is a large summand of $R$.

(iii) $\Rightarrow$ (i): First, we note that $\operatorname{dim} \operatorname{End}(V)=p+\operatorname{dim} \operatorname{End}(R)$ for $V$ in the open sheet. For, consider the representations $H \oplus R$, where $H$ is the direct sum of $p$ homogeneous simple regular modules which are pairwise non-isomorphic. Then clearly $\operatorname{dim} \operatorname{Ext}(H \oplus R, H \oplus R)=p$. Thus we have a $p$-parameter family of orbits of codimension $p$ in $\mathbb{A}[\Gamma, \mathbf{d}]$, so some, and hence all, of these representations belong to the open sheet. But again we see immediately that End $(H \oplus R)$ has dimension $p+\operatorname{dim} \operatorname{End}(R)$. 
But an easy calculation shows that for the representations in (iii), the dimension of the endomorphism ring is precisely $p+\operatorname{dim} \operatorname{End}(R)$.

Corollary 3.6. Let $\Gamma$ be a tame quiver. Let $\operatorname{dim} V=\mathbf{d}$. Then $V$ belongs to the open sheet in $\mathbb{A}[\Gamma, \mathbf{d}]$ if and only if for any direct decomposition $V=V^{\prime} \oplus V^{\prime \prime}$, we have $\operatorname{Ext}\left(V^{\prime}, V^{\prime \prime}\right)=0$.

Proof. According to 2.2, one implication is true for any $I$. Now assume, $V$ satisfies this extension condition. Let $V=P \oplus Q \oplus R$ with $P$ preprojective, $Q$ preinjective and $R$ regular. Let $\operatorname{dim} R=p \mathbf{b}+\sum_{i \in I} p_{i} \mathbf{e}_{i}$ be the canonical decomposition. If $P \oplus Q \neq 0$, then according to 3.1 , we have $p=0$. But for any indecomposable module $U$ which is preprojective, or preinjective, or regular without having a complete $C^{+}$-orbit of simple regular modules as regular composition factors, we have $\operatorname{Ext}(U, U)=0$. Thus, if we write $V$ as direct sum of indecomposable representations, we immediately see that $\operatorname{Ext}(V, V)=0$, and therefore $V$ belongs to an open orbit (2.4), thus to the open sheet.

Thus, we may assume $V$ is regular. But then $\mathbf{d} \in \mathfrak{D}_{r}$, and we can apply 3.5 .

Remark. If $\Gamma$ is wild, the corresponding assertion is no longer true. Consider for example the quiver

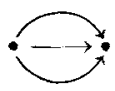

and the dimension type $\mathbf{d}=(2,2)$. Then the representations $V$

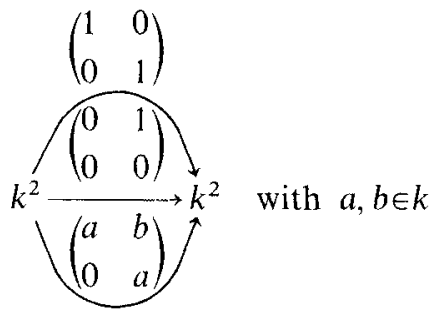

are indecomposable and therefore satisfy the property. $\operatorname{Ext}\left(V^{\prime}, V^{\prime \prime}\right)=0$ for $V$ $=V^{\prime} \oplus V^{\prime \prime}$, trivially. However, they do not belong to the open sheet, since $\operatorname{dim} \operatorname{End}(V)=2$, whereas there are representations $W$ of dimension type $(2,2)$ with $\operatorname{End}(W)=k$.

It can be shown quite easily that the equivalence given in the corollary characterises the quivers of finite and tame representation type.

\section{The Construction Process of the Invariants} Recall that for any $\mathbf{d} \in \mathfrak{D}=\mathbb{Q}^{n}$, the characters of the group $G(\mathbf{d})=\prod_{i=1}^{n} G L\left(d_{i}\right)$ are
of the form $\chi_{\lambda}$,

$$
\chi_{\lambda}: g=\left(g_{i}\right) \mapsto \prod_{i}\left(\operatorname{det} g_{i}\right)^{\lambda_{1}},
$$


with $\lambda=\left(\lambda_{i}\right) \in\left(\mathbb{Z}^{n}\right)^{*} \subset \mathfrak{D}^{*}$. Also recall that a regular function $f \in k[\Gamma, \mathbf{d}]$ is called a semi-invariant of weight $\lambda$ provided

$$
g * f=\chi_{\lambda}(g) \cdot f
$$

and that we denote by $k[\Gamma, \mathbf{d}]_{\lambda}$ the set of regular semi-invariants of weight $\lambda$.

We assume now that $\mathbf{d} \in \mathfrak{D}_{r}$. In particular, $\partial \mathbf{d}=0$. We want to consider a certain matrix with coefficients in the polynomial ring $k[\Gamma, \mathbf{d}][S, T]$ in the $m+2$ variables $X_{x s i}, S, T$. This matrix will be a square matrix precisely because of the condition $\partial \mathbf{d}=0$, so that we can compute its determinant $F\left(X_{x s t} S, T\right)$ $\in k[T, \mathbf{d}][S, T]$. By construction, this will be a homogeneous polynomial in the variables $S, T$, and it will belong to the weight space $k[\Gamma, \mathbf{d}]_{c}[S, T]$.

Also, the polynomials $F=F_{\mathrm{d}} \in k[\Gamma, \mathbf{d}][S, T]$ will have the following multiplicative property: Assume there is given a representation $\left(\varphi_{\alpha s}\right) \in \mathbb{A}[\Gamma, \mathbf{d}]$ which is reducible, say $\mathbf{d}=\mathbf{d}^{\prime}+\mathbf{d}^{\prime \prime}$, and for every arrow $i \stackrel{\alpha}{\longrightarrow}$, we have $\varphi_{\alpha s t}=0$ for $1 \leqq s \leqq d_{i}^{\prime}$ and $d_{j}^{\prime}<t \leqq d_{j}^{\prime}+d_{j}^{\prime \prime}=d_{j}$. Let $\varphi_{\alpha s t}^{\prime}=\varphi_{\alpha s t}$ for $1 \leqq s \leqq d_{i}^{\prime}, 1 \leqq t \leqq d_{j}^{\prime}$, and $\varphi_{\alpha s t}^{\prime \prime}$ $=\varphi_{\alpha, d_{i}^{\prime}+s, d_{i}^{\prime}+t}$, for $1 \leqq s \leqq d_{i}^{\prime \prime}, 1 \leqq t \leqq d_{j}^{\prime \prime}$, where $i \stackrel{\alpha}{\longrightarrow} j$. In this way we get representations $\left(\varphi_{\alpha s t}^{\prime}\right) \in \mathbb{A}\left[\Gamma, \mathbf{d}^{\prime}\right],\left(\varphi_{\alpha s t}^{\prime \prime}\right) \in \mathbb{A}\left[\Gamma, \mathbf{d}^{\prime \prime}\right]$, and a short exact sequence

$$
0 \rightarrow\left(\varphi_{\alpha s t}^{\prime}\right) \rightarrow\left(\varphi_{\alpha s t}\right) \rightarrow\left(\varphi_{\alpha s t}^{\prime \prime}\right) \rightarrow 0 .
$$

The multiplicative property of the polynomials $F_{\mathrm{d}}$ asserts that then

$$
F_{\mathbf{d}}\left(\varphi_{\alpha s t}, S, T\right)=F_{\mathbf{d}^{\prime}}\left(\varphi_{\alpha s t}^{\prime}, S, T\right) \cdot F_{\mathbf{d}^{\prime \prime}}\left(\varphi_{\alpha, s t}^{\prime \prime}, S, T\right) .
$$

In particular, given $\left(\varphi_{\alpha s t}^{\prime}\right) \in \mathbb{A}\left[\Gamma, \mathbf{d}^{\prime}\right]$ and $\left(\varphi_{\alpha s t}^{\prime \prime}\right) \in \mathbb{A}\left[\Gamma, \mathbf{d}^{\prime \prime}\right]$, we see that for the direct sum representation $\left(\varphi_{x s t}\right)=\left(\varphi_{x s t}^{\prime}\right) \oplus\left(\varphi_{\alpha s t}^{\prime \prime}\right)$ in $\mathbb{A}\left[\Gamma, \mathbf{d}^{\prime}+\mathbf{d}^{\prime \prime}\right]$, we have the equality $(*)$.

Besides this, we will construct an affine embedding

$$
\mathbb{A}[\Delta, p] \rightarrow \mathbb{A}[\Gamma, \mathbf{d}],
$$

where $\mathbf{d}=p \mathbf{h}+\sum_{i \in I} p_{i} \mathbf{e}_{i}$ is the canonical decomposition of $\mathbf{d}$, such that the union of the orbits containing elements in the image contains a dense open subset.

In fact, we will define first an affine embedding

$$
M: \mathbb{A}[\Sigma,(p, p)] \rightarrow \mathbb{A}[\Gamma, p \mathbf{h}]
$$

such that the image contains representatives of all homogeneous representations of dimension type $p \mathbf{h}$. Now fix a regular representation $R$ of dimension type $\Sigma p_{i} \mathbf{e}_{i}$ with $\operatorname{Ext}(R, R)=0$, such a representation exists according to 3.4 and is unique up to isomorphism. The affine embedding $\mathbb{A}[\Sigma,(p, p)] \rightarrow \mathbb{A}[\Gamma, \mathbf{d}]$ then will be given by $(\Phi, \Psi) \mapsto M(\Phi, \Psi) \oplus R$. Calculating dimensions, it is easy to see that the image of the regular map

$$
\mathbb{A}[\Sigma,(p, p)] \times G(\mathbf{d}) \rightarrow \mathbb{A}[\Gamma, \mathbf{d}],
$$

given by $(\Phi, \Psi) \times g \mapsto g *(M(\Phi, \Psi) \oplus R)$ is dense in $\mathbb{A}[\Gamma, \mathbf{d}]$. For, we see that the image contains the $p$-parameter family of all representations of the form $H \oplus R$, 
with $H$ the direct sum of $p$ homogeneous, simple regular, and pairwise nonisomorphic modules, and the orbit dimension of any such $H \oplus R$ is just $\operatorname{dim} \mathbb{A}[\Gamma, \mathbf{d}]-p($ see 3.5$)$.

We want to show that $(* *) \quad F(M(\Phi, \Psi) \oplus R)=S^{p_{0}}(S-T)^{p_{1}} T^{p_{\infty}} \operatorname{det}(S \Phi+T \Psi)$, for some choice of the representation $R$. In order to see this, it will be sufficient to show that

$$
\begin{gathered}
F(M(\Phi, \Psi))=\operatorname{det}(S \Phi+T \Psi), \\
F\left(E_{0}\right)=S, \quad F\left(E_{1}\right)=S-T, F\left(E_{\infty}\right)=T, \\
F\left(E_{i}\right)=1 \quad \text { for } i \in I \backslash\{0,1, \infty\},
\end{gathered}
$$

using the multiplicative property of the polynomials $F$, where $E_{0}, E_{1}, E_{\infty}$ are suitable simple regular representations which have to be choosen in each case separately.

Note that it follows from $(* *)$ that for a representation $\left(\varphi_{\alpha s t}\right)$ isomorphic to $M(\Phi, \Psi) \oplus R$, say $\left(\varphi_{\alpha s t}\right)=g *(M(\Phi, \Psi) \oplus R)$ with some $g \in G(\mathbf{d})$, we have

$$
\begin{aligned}
F\left(\varphi_{\alpha s t}, S, T\right) & =\prod\left(\operatorname{det} g_{i}\right)^{\partial_{2}} F(M(\Phi, \Psi) \oplus R, S, T) \\
& =\prod\left(\operatorname{det} g_{i}\right)^{\partial_{2}} S^{p_{0}}(S-T)^{p_{1}} T^{p_{\infty}} \operatorname{det}(S \Phi+T \Psi) .
\end{aligned}
$$

This shows that on a dense subset of $\mathbb{A}[\Gamma, \mathbf{d}]$, the polynomial $F\left(X_{\alpha s t}, S, T\right)$ is divisible by $S^{p_{0}}(S-T)^{p_{1}} T^{p_{\infty}}$, thus

$$
\left.F\left(X_{\alpha s t}\right), S, T\right)=S^{p_{0}}(S-T)^{p_{1}} T^{p_{\infty}} \sum_{i=0}^{p} f_{i}\left(X_{\alpha s t}\right) S^{i} T^{p-i}
$$

for some polynomials $f_{i}\left(X_{\alpha s t}\right) \in k[\Gamma, \mathbf{d}]$, and, in fact, in $k[\Gamma, \mathbf{d}]_{\partial}$. And it follows from $(* *)$ that

$$
\sum_{i=0}^{p} f_{i}(M(\Phi, \Psi) \oplus R) S^{i} T^{p-i}=\operatorname{det}(S \Phi+T \Psi) .
$$

We will always denote by $I$ the $p \times p$ identity matrix. For $\Phi \in \mathbb{A}[\Delta, p]$, let $M(\Phi)=M(\Phi, I) \in \mathbb{A}[\Gamma, p \mathbf{h}]$. In this way, we obtain an embedding $\Phi \mapsto M(\Phi) \oplus R$

$$
\mathbb{A}[\Delta, p] \hookrightarrow \mathbb{A}[\Gamma, \mathbf{d}] .
$$

For $S=1$, the equality

$$
\sum_{i=0}^{p} f_{i}(M(\Phi) \oplus R) T^{p-i}=\operatorname{det}(\Phi+T)
$$

shows that the rational functions

$$
\frac{f_{i}(M(\Phi) \oplus R)}{f_{0}(M(\Phi) \oplus R)}, \quad 1 \leqq i \leqq p
$$

are (up to the sign) the coefficients of the characteristic polynomial $\operatorname{det}(\Phi-T)$ of 
$\Phi$. In particular, they are algebraically independent.

Thus, consider the rational functions

$$
\frac{f_{i}\left(X_{\alpha s t}\right)}{f_{0}\left(X_{\alpha s t}\right)}, \quad 1 \leqq i \leqq p
$$

on $\mathbb{A}[\Gamma, \mathbf{d}]$. Note that they belong to the field $I(\Gamma, \mathbf{d})$ of rational invariants of $\mathbb{A}\left[\Gamma^{\prime}, \mathbf{d}\right]$ under $G(\mathbf{d})$, since all $f_{i}\left(X_{\alpha s t}\right)$ belong to the same weight space $k\left[X_{\alpha s t}\right]_{\partial}$. Clearly, they are algebraically independent. Since the representations isomorphic to representations of the form $M(\Phi) \oplus R$, with $\Phi \in \mathbb{A}[4, p]$ are dense in $\mathbb{A}[\Gamma, \mathbf{d}]$, it follows that these rational functions also generate $I(\Gamma, \mathbf{d})$.

Theorem 4.1. Let $\mathbf{d} \in \mathcal{D}_{r}$, with canonical decomposition $\mathbf{d}=p \mathbf{h}+\sum_{i \in I} p_{i} \mathbf{e}_{i}$. Then there exist semi-invariants $f_{0}, \ldots, f_{p} \in k[\Gamma, \mathbf{d}]_{e}$ such that the elements $\frac{f_{1}}{f_{0}}, \ldots, \frac{f_{p}}{f_{0}}$ form a
transcendence basis of $I(\Gamma, \mathbf{d})$.

\section{The Different Tame Cases}

In dealing with the different tame quivers, we will restrict our attention to one particular orientation. Note that we can obtain any other orientation by a sequence of elementary changes of orientation with corresponding reflection functors [2], and one can determine the change of semi-invariants under such a change of orientation, see V. Kac [10].

For a given orientation, we also list the defect $\partial$ by writing down $\partial_{i}$ at the $i$-th position. Also, we denote by $I$ the $p \times p$ identity matrix.

Case $\tilde{\mathbf{A}}_{u, v}, u \geqq v \geqq 1$. This is the following quiver

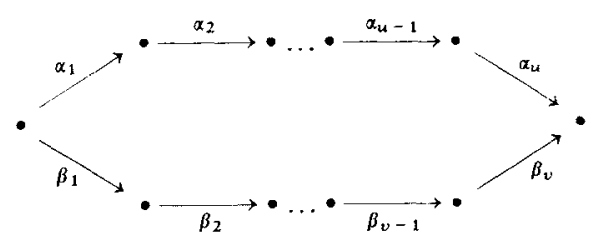

where the $\alpha_{i}, \beta_{i}$ both are names of the arrows, and, for a given representation, will also denote the corresponding linear map. The defect is given by

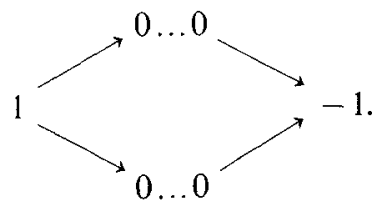

If $\mathbf{d}$ is a dimension type with $\partial(\mathbf{d})=0$, let

$$
F_{\mathbf{d}}\left(X_{\alpha s t}, S, T\right):=\operatorname{det}\left(S \alpha_{1} \ldots \alpha_{u}+T \beta_{1} \ldots \beta_{v}\right),
$$


where $\alpha_{1} \ldots \alpha_{u}$ stands for the composition of maps (or matrices). Since $\partial(\mathbf{d})=0$, the two matrices $\alpha_{1} \ldots \alpha_{u}$ and $\beta_{1} \ldots \beta_{v}$ (which always are of the same size) are square matrices. Note that $F_{\mathrm{d}}$ is homogeneous in $S, T$, but it is not homogeneous in the other variables, except in the case $u=v$.

For the dimension type $\mathbf{d}=p \mathbf{h}$, where $\mathbf{h}=\left(\begin{array}{lll}1 & 1 & \ldots 1 \\ 1 \ldots 1\end{array}\right)$, we define the affine embedding $M: \mathbb{A}[\Sigma,(p, p)] \rightarrow \mathbb{A}[\Gamma, p \mathbf{h}]$ by the rule that $(\Phi, \Psi) \in \mathbb{A}[\Sigma,(p, p)]$ is sent to

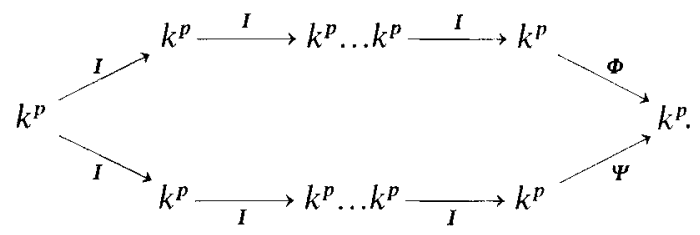

Then $F(M(\Phi, \Psi), S, T)=\operatorname{det}(S \Phi+T \Psi)$, and for

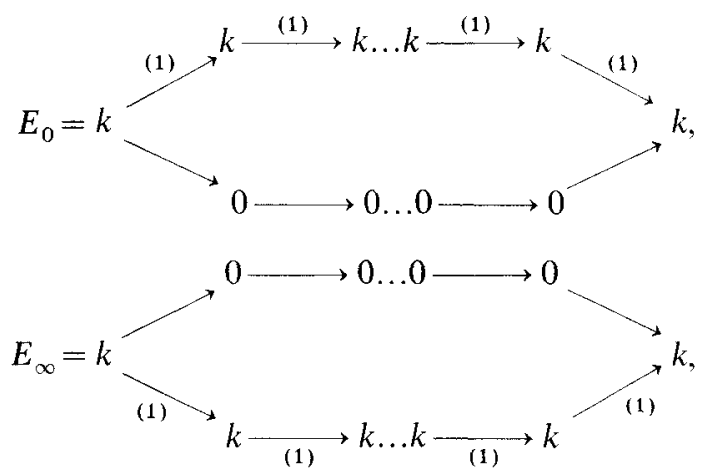

we have $F\left(E_{0}, S, T\right)=S, F\left(E_{\infty}, S, T\right)=T$.

Case $\tilde{\mathbf{D}}_{n}, n \geqq 4$. This is the quiver:

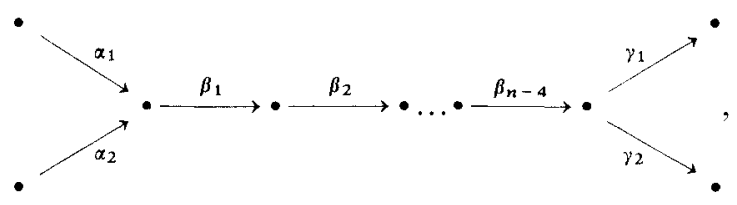

its defect is given by

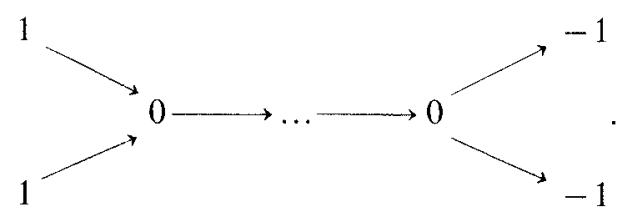


Denote $\beta:=\beta_{1} \ldots \beta_{n-4}$, and for $\mathbf{d}$ with $\partial(\mathbf{d})=0$, let

$$
F_{\mathrm{d}}\left(X_{\alpha s t}, S, T\right):=\left(\frac{S \alpha_{1} \beta \gamma_{1}}{\alpha_{2} \beta \gamma_{1}} \mid \frac{T \alpha_{1} \beta \gamma_{2}}{\alpha_{2} \beta \gamma_{2}}\right) .
$$

We define $M: \mathbb{A}[\Sigma,(p, p)] \rightarrow \mathbb{A}[\Gamma, p \mathbf{h}]$ by the rule that $M(\Phi, \Psi)$ is

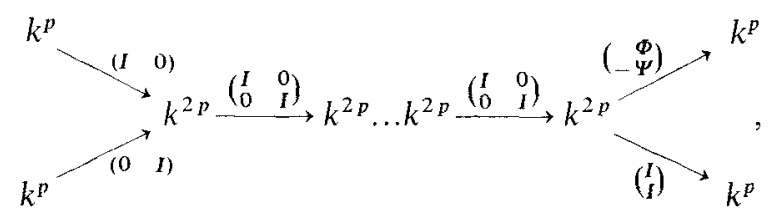

Then $F(M(\Phi, \Psi), S, T)=\operatorname{det}(S \Phi+T \Psi)$.

For the representations

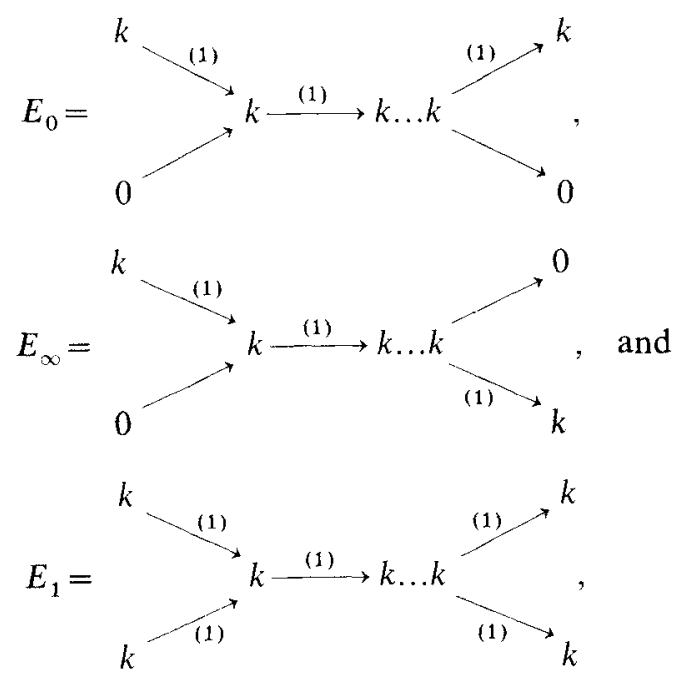

we have $F\left(E_{0}, S, T\right)=S, F\left(E_{\infty}, S, T\right)=T, F\left(E_{1}, S, T\right)=S-T$.

Let us pause for a moment and consider also another orientation in the case of type $\tilde{\mathbf{D}}_{4}$, namely

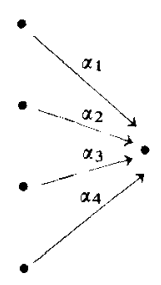

In this case, the representations without simple projective direct summands are given by a vector space with four subspaces, thus one speaks of the four 
subspace problem $[12,9]$. For this case, the notion of defect was introduced in [9] by Gelfand and Ponomarev; it is given by

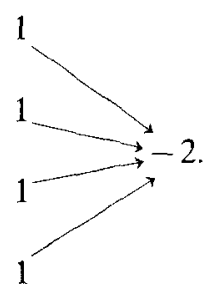

Given $\mathbf{d}$ with $\partial(\mathbf{d})=0$, the polynomial

$$
F_{d}\left(X_{a s t}, S, T\right):=\operatorname{det}\left(\begin{array}{cc}
S \alpha_{1} & T \alpha_{1} \\
\alpha_{2} & 0 \\
\alpha_{3} & \alpha_{3} \\
0 & \alpha_{4}
\end{array}\right)
$$

gives us $p+1$ semi-invariants $f_{0}, \ldots, f_{p} \in k[\Gamma, \mathbf{d}]_{\gamma}[S, T]$, where $\mathbf{d}=p \mathbf{h}+\Sigma p_{i} \mathbf{e}_{i}$ is the canonical decomposition.

In particular, consider $\mathbf{d}=\left(\begin{array}{l}1 \\ 1 \\ 1 \\ 1\end{array}\right)$, and let $\alpha_{i}=\left(x_{i}, y_{i}\right)$ with coordinate functions $X_{i}, Y_{i}$ for $1 \leqq i \leqq 4$. Then $F_{\mathbf{d}}=f_{0} T+f_{1} S$, with

$$
\begin{aligned}
& f_{0}=X_{1} X_{2} Y_{3} Y_{4}-X_{1} Y_{2} X_{3} Y_{4}-Y_{1} X_{2} Y_{3} X_{4}+Y_{1} Y_{2} X_{3} X_{4} \\
& f_{1}=X_{1} Y_{2} X_{3} Y_{4}-X_{1} Y_{2} Y_{3} X_{4}-Y_{1} X_{2} X_{3} Y_{4}+Y_{1} X_{2} Y_{3} X_{4}
\end{aligned}
$$

Given an element $\left(x_{i}, y_{i}\right)_{1 \leqq i \leqq 4} \in \mathbb{A}[\Gamma, \mathbf{d}]$ with $\left(x_{i}, y_{i}\right) \neq(0,0)$ for all $i$, this determines a quadrupel of points $P_{i}=\left(x_{i}, y_{i}\right)$ in the projective line $\mathbb{P}_{1}$, and $\frac{f_{1}\left(x_{i}, y_{i}\right)}{f_{0}\left(x_{i}, y_{i}\right)}$
is just (the negative of the cross ratio

$$
\frac{\frac{x_{1}}{y_{1}}-\frac{x_{2}}{y_{2}}}{\frac{x_{1}}{y_{1}}-\frac{x_{4}}{y_{4}}}: \frac{\frac{x_{3}}{y_{3}}-\frac{x_{2}}{y_{2}}}{\frac{x_{3}}{y_{3}}-\frac{x_{4}}{y_{4}}}
$$

of the four points $P_{1}, \ldots, P_{4}$.

The regular representations of $\Gamma$ all are given by a vector space with four subspaces, and our construction of rational invariants in this case means that we compute a full set of rational invariants for the position of four subspaces in an arbitrary projective space.

Case $\tilde{\mathbf{E}}_{6}$. We consider the quiver $\Gamma$ with the following orientation 


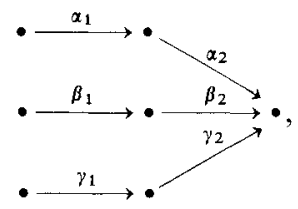

the corresponding defect $\partial$ being

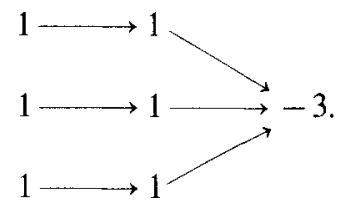

For d with $\partial(\mathbf{d})$, let

$$
F_{\mathbf{d}}\left(X_{\alpha s t}, S, T\right):=\operatorname{det}\left(\begin{array}{ccc}
0 & S \alpha_{1} \alpha_{2} & T \alpha_{1} \alpha_{2} \\
\alpha_{2} & 0 & 0 \\
\beta_{1} \beta_{2} & 0 & \beta_{1} \beta_{2} \\
0 & \beta_{2} & 0 \\
\gamma_{1} \gamma_{2} & \gamma_{1} \gamma_{2} & 0 \\
0 & 0 & \gamma_{2}
\end{array}\right)
$$

We define $M: \mathbb{A}[\Sigma,(p, p)] \rightarrow \mathbb{A}[\Gamma, p \mathbf{h}]$ by the rule that $M(\Phi, \Psi)$ is

$$
\begin{aligned}
& k^{p \stackrel{(\Phi \Psi}{\longrightarrow}} k^{2 p} \underbrace{\left(\begin{array}{lll}
I & 0 & 0 \\
0 & I & 0
\end{array}\right)} \\
& k^{p \stackrel{(I I)}{\longrightarrow}} k^{2 p} \stackrel{\left(\begin{array}{lll}
0 & I & 0 \\
0 & 0 & I
\end{array}\right)}{\stackrel{\left(\begin{array}{lll}
I & 0 & 0 \\
0 & 0 & I
\end{array}\right)}{\longrightarrow}} k^{3 p}, \\
& k^{p} \stackrel{(I I)}{\longrightarrow} k^{2 p}
\end{aligned}
$$

Then $F(M(\Phi, \Psi), S, T)=\operatorname{det}(S \Phi+T \Psi)$.

For the representations

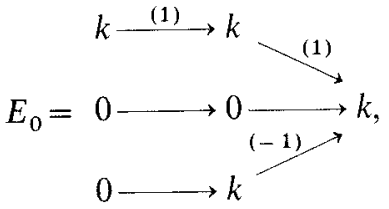

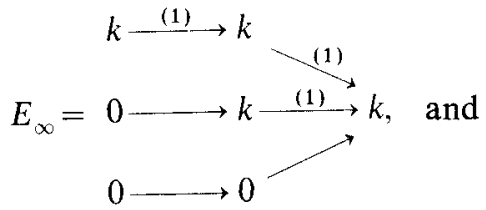




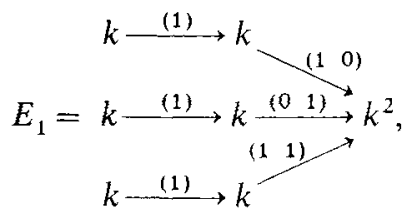

we have $F\left(E_{0}, S, T\right)=S, F\left(E_{\infty}, S, T\right)=T, F\left(E_{1}, S, T\right)=S-T$.

Case $\tilde{\mathbf{E}}_{7}$. We consider the quiver $\Gamma$ with the following orientation

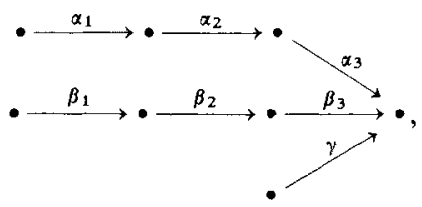

the corresponding defect $\partial$ being

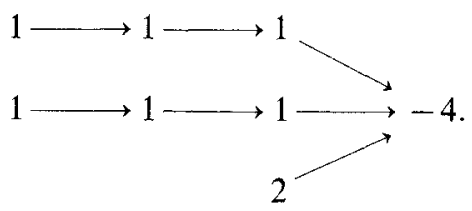

For any dimension type $\mathbf{d}$ with $\partial(\mathbf{d})=0$, let

$$
F_{d}\left(X_{\alpha s t}, S, T\right):=\operatorname{det}\left(\begin{array}{cccc}
0 & 0 & S \alpha_{1} \alpha_{2} \alpha_{3} & T \alpha_{1} \alpha_{2} \alpha_{3} \\
\alpha_{2} \alpha_{3} & 0 & 0 & 0 \\
0 & \alpha_{3} & 0 & 0 \\
\beta_{1} \beta_{2} \beta_{3} & \beta_{1} \beta_{2} \beta_{3} & 0 & 0 \\
0 & 0 & \beta_{2} \beta_{3} & 0 \\
0 & 0 & 0 & \beta_{3} \\
\gamma & 0 & 0 & \gamma \\
0 & \gamma & \gamma & 0
\end{array}\right) .
$$

We define $M: \mathbb{A}[\Sigma,(p, p)] \rightarrow \mathbb{A}[\Gamma, p \mathbf{h}]$ by the rule that $M(\Phi, \Psi)$ is

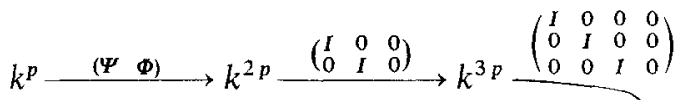

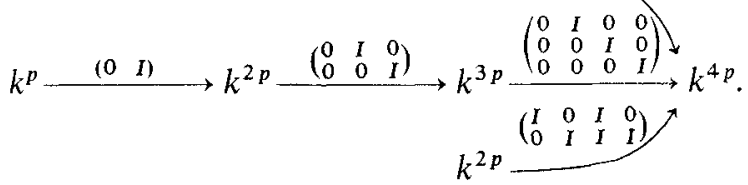

Then $F(M(\Phi, \Psi), S, T)=\operatorname{det}(S \Phi+T \Psi)$, and for

$$
E_{0}=0 \longrightarrow \text { 는 }
$$




$$
\begin{aligned}
& k-\stackrel{(1)}{\longrightarrow} k-\stackrel{(10)}{\longrightarrow} k^{2} \quad\left(\begin{array}{ll}
1 & 0 \\
0 & 1
\end{array}\right)
\end{aligned}
$$

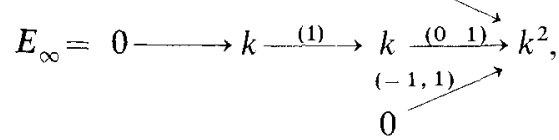

$$
\begin{aligned}
& k \stackrel{(1)}{\longrightarrow} k \stackrel{(1)}{\longrightarrow} k \\
& E_{1}=k \stackrel{(1)}{\longrightarrow} k \stackrel{(1)}{\longrightarrow} \underset{k}{k \stackrel{(0 \stackrel{1}{(-1} 1)}{\longrightarrow}} k^{2} .
\end{aligned}
$$

We have $F\left(E_{0}, S, T\right)=S, F\left(E_{\infty}, S, T\right)=T, F\left(E_{1}, S, T\right)=S-T$.

Case $\tilde{\mathbf{E}}_{8}$. We consider the quiver $\Gamma$ with the following orientation

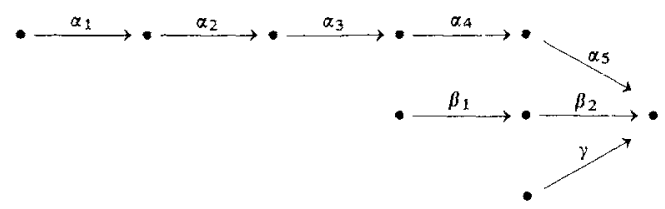

the corresponding defect being given by

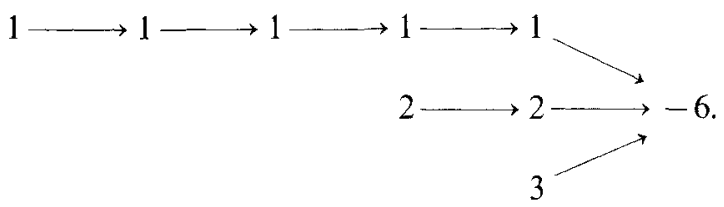

For any d will $\partial(\mathbf{d})=0$, let $F_{\mathbf{d}}\left(X_{\alpha s t}, S, T\right)$ be the determinant of the following matrix

$$
\left(\begin{array}{cccccc}
0 & 0 & 0 & S \alpha_{12345} & 0 & T \alpha_{12345} \\
0 & 0 & 0 & \alpha_{2345} & \alpha_{2345} & \alpha_{2345} \\
\alpha_{345} & 0 & 0 & 0 & 0 & 0 \\
0 & \alpha_{45} & 0 & 0 & 0 & 0 \\
0 & 0 & \alpha_{5} & 0 & 0 & 0 \\
\beta_{12} & \beta_{12} & 0 & 0 & 0 & 0 \\
0 & 0 & 0 & \beta_{12} & 0 & \beta_{12} \\
0 & 0 & 0 & \beta_{2} & 0 & 0 \\
0 & 0 & 0 & 0 & \beta_{2} & 0 \\
\gamma & 0 & 0 & 0 & \gamma & 0 \\
0 & \gamma & \gamma & \gamma & 0 & 0 \\
0 & 0 & 0 & \gamma & 0 & \gamma
\end{array}\right),
$$

where $\alpha_{i \ldots j}=\alpha_{i} \cdot \alpha_{i+1} \ldots \alpha_{j}, \beta_{12}=\beta_{1} \cdot \beta_{2}$. 
We define $M: \mathbb{A}[\Sigma,(p, p)] \rightarrow \mathbb{A}[\Gamma, p \mathbf{h}]$ by the rule that $M(\Phi, \Psi)$ is

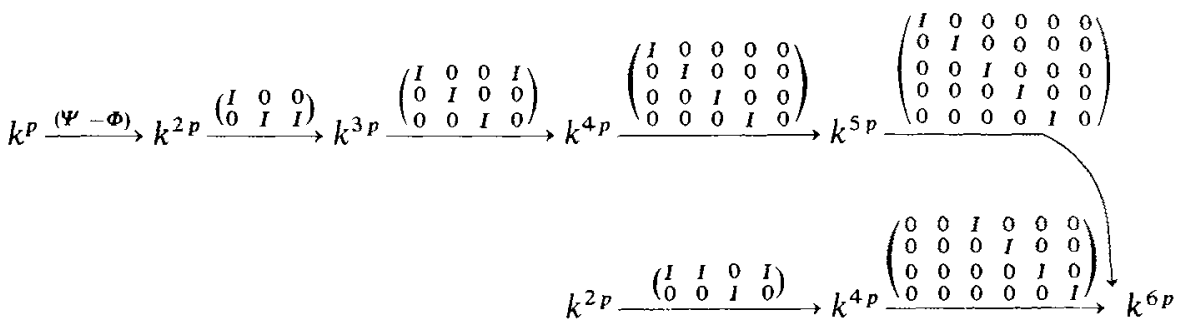

$$
\begin{aligned}
& \left.\left(\begin{array}{llllll}
1 & 0 & 0 & 0 & 0 & 0 \\
0 & I & 0 & 0 & I & 0 \\
0 & 0 & 0 & 0 & 0 & I
\end{array}\right)\right)
\end{aligned}
$$

Then it follows that $F(M(\Phi, \Psi), S, T)=\operatorname{det}(S \Phi+T \Psi)$. For the simple regular representations

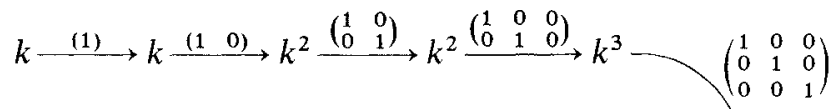

$$
\begin{aligned}
& E_{1}=\quad k \stackrel{\left(\begin{array}{ll}
0 & 1
\end{array}\right)}{\longrightarrow} k^{2} \stackrel{\left(\begin{array}{lll}
0 & 1 & 0 \\
0 & 0 & 1
\end{array}\right) \downarrow}{\longrightarrow} k^{3}, \\
& \left(\begin{array}{lll}
-1 & 1 & 1
\end{array}\right) \\
& \left.k \stackrel{(1)}{\longrightarrow} k \stackrel{(1)}{\longrightarrow} k \stackrel{(1}{\longrightarrow}{ }^{0}\right) k^{2} \stackrel{\left(\begin{array}{ll}
1 & 0 \\
0 & 1
\end{array}\right)}{\longrightarrow} k^{2} \\
& \left(\begin{array}{ll}
1 & 0 \\
0 & 1
\end{array}\right) \\
& E_{\infty}=\quad 0 \longrightarrow k \stackrel{(01)}{\longrightarrow} k^{2} \text {, and } \\
& k \stackrel{(1)}{\longrightarrow} k \stackrel{(1)}{\longrightarrow} k \stackrel{(1)}{\longrightarrow} k \stackrel{(1)}{\longrightarrow} k \\
& \left(\begin{array}{ll}
1 & 0
\end{array}\right. \\
& E_{1}= \\
& k \stackrel{(1)}{\longrightarrow} k \stackrel{(011)}{\longrightarrow} k^{2} \text {, } \\
& k
\end{aligned}
$$

we have $F\left(E_{0}, S, T\right)=S, F\left(E_{\infty}, S, T\right)=T$, and $F\left(E_{1}, S, T\right)=S-T$.

\section{The Quotient Map}

Now, let $k$ be an algebraically closed field. We assume throughout this section that $\Gamma$ is a tame quiver and $\mathbf{d} \in \mathfrak{D}_{r}$, say with canonical decomposition $\mathbf{d}=p \mathbf{h}$ 
$+\sum_{i \in I} p_{i} \mathbf{e}_{i}$. We have constructed in the previous sections polynomial semi-invariants $f_{0}, \ldots, f_{p}$ which form a basis of $k[\Gamma, \mathbf{d}]_{\hat{\theta}}$, where $\partial$ is the defect. Let us consider the corresponding rational map

$$
f=\left(f_{0}: \ldots, f_{\mathbf{p}}\right): \mathbb{A}[\Gamma, \mathbf{d}] \rightarrow \mathbb{P}_{p},
$$

where $\mathbb{P}_{p}$ is the $p$-dimensional projective space. It is clear that this map $f$ is constant on $G(\mathbf{d})$-orbits and we will see that we may consider $f$ as a quotient map.

Theorem 6.1. The rational map $f: \mathbb{A}[\Gamma, \mathbf{d}] \rightarrow \mathbb{P}_{p}$ is regular precisely in those points $\left(\varphi_{\text {ast }}\right) \in \mathbb{A}[\Gamma, \mathbf{d}]$ which are regular representations.

In particular, the set of regular representations is open; it is the complement of the zero set $V\left(f_{0}, \ldots, f_{p}\right)$.

Proof. By definition,

$$
F\left(\varphi_{\alpha s t}, S, T\right)=S^{p_{0}}(S-T)^{p_{1}} T^{p_{\infty}} \sum_{i=0}^{p} f_{i}\left(\varphi_{\alpha s t}\right) S^{i} T^{p-i},
$$

thus $\left(\varphi_{\alpha s t}\right) \in V\left(f_{0}, \ldots, f_{p}\right)$ if and only if $F\left(\varphi_{x s t}, S, T\right)=0$. If $\left(\varphi_{\alpha s t}\right)$ is not regular, then it has a non-zero direct summand of non-zero defect. But this means that for some base change $g \in G(\mathbf{d})$, the polynomial $F\left(g *\left(\varphi_{\alpha s t}\right), S, T\right)$ is the determinant of a matrix which is (up to permutations of rows and columns) of the form

$$
\left(\begin{array}{c|c}
* & 0 \\
\hline 0 & *
\end{array}\right)
$$

with non-square diagonal blocks. This shows that $F\left(g *\left(\varphi_{\alpha s t}\right), S, T\right)=0$, and therefore also $F\left(\varphi_{x s t}, S, T\right)=0$.

On the other hand, let $\left(\varphi_{\alpha s t}\right)$ be regular. Using the multiplicative property of determinants on extensions, one sees that one can restrict to $\left(\varphi_{\alpha s t}\right)$ being simple regular. But then case by case inspection as in Section 5 shows that always $F\left(\varphi_{a s t}, S, T\right) \neq 0$.

In particular, the map $f$ is regular on the open sheet, since this consists entirely of regular modules. Let us denote the open sheet by $\mathbf{A}[\Gamma, \mathbf{d}]^{\circ}$, and let us consider the restriction of $f$ to $\mathbb{A}[\Gamma, \mathbf{d}]^{0}$.

Theorem 6.2. The regular map $f: \mathbb{A}[\Gamma, \mathbf{d}]^{\circ} \rightarrow \mathbb{P}_{p}$ is surjective. It is injective on the set of $G$-orbits except perhaps on the set of $G$-orbits contained in the intersection of $\mathbb{A}[\Gamma, \mathbf{d}]^{0}$ with the three hyperplanes $V\left(f_{0}\right), V\left(f_{p}\right)$ and $V\left(\sum_{i=0}^{p} f_{i}\right)$.

Proof. Let us first prove the surjectivity of $f: \mathbb{A}[\Gamma, \mathbf{d}] \rightarrow \mathbb{P}_{p}$. Define an embedding

$$
N:\left(\mathbb{A}^{2} \backslash\{(0,0)\}\right)^{p \rightarrow} \rightarrow \mathbb{A}[\Sigma,(p, p)]
$$

by

$$
N\left(\left(a_{1}, b_{1}\right), \ldots,\left(a_{p}, b_{p}\right)\right)=\left(\left(\begin{array}{cc}
a_{1} & 0 \\
0 & a_{p}
\end{array}\right),\left(\begin{array}{cc}
b_{1} & 0 \\
0 & b_{p}
\end{array}\right)\right),
$$

for $\left(a_{i}, b_{i}\right) \in \mathbb{A}^{2} \backslash\{(0,0)\}$ and note that all the representations $M N\left(\left(a_{i}, b_{i}\right)_{i}\right) \oplus R$ are regular. It is clear that the composition with $f$ factors over $\left(\mathbb{P}_{1}\right)^{p}$, say 


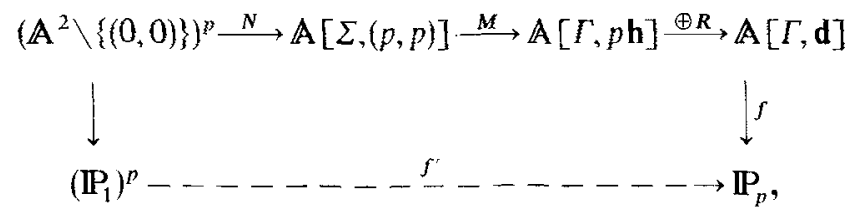

and that the image of $f^{\prime}$ is dense in $\mathbb{P}_{p}$. Since $\left(\mathbb{P}_{1}\right)^{p}$ is a projective variety, the image of $f^{\prime}$ has to be closed, and therefore equal to $\mathbb{P}_{p}$. This shows that $f$ is surjective.

Using again the multiplicativity of $F$ on extensions, one sees immediately, that for any regular representation $\left(\varphi_{x s s}\right)$ we find a representation $\left(\psi_{\alpha s s}\right)$ in $\mathbb{A}[\Gamma, \mathbf{d}]^{0}$ with $f\left(\varphi_{\text {ass }}\right)=f\left(\psi_{\text {ast }}\right)$. Thus, also the restriction of $f$ to $\mathbb{A}[\Gamma, \mathbf{d}]^{0}$ is surjective. Next, we note that a representation $\left(\varphi_{a s t}\right)$ belongs to $V\left(f_{0}\right)$ if and only if $S^{p_{0}+1}$ is a factor of $F\left(\varphi_{x s 1}, S, T\right)$ and this happens if and only if in a composition series of $\left(\varphi_{\alpha s i}\right)$, the simple regular representation $E_{0}$ appears with multiplicity at least $p_{0}+1$. Similarly, $\left(\varphi_{x, s}\right)$ belongs to $V\left(f_{x}\right)$ if and only if $T^{p_{\omega}+1}$ is a factor of $F\left(\varphi_{x s t}, S, T\right)$, if and only if $E_{\infty}$ appears with multiplicity at least $p_{x}$ +1 , and finally $\left(\varphi_{x s t}\right)$ belongs to $V\left(\sum_{i=0}^{p} f_{i}\right)$ if and only if $T^{p_{1}+1}$ is a factor of $F\left(\varphi_{\alpha s t}, S, T\right)$, if and only if $E_{1}$ appears with multiplicity at least $p_{1}+1$. Thus, if $\left(\varphi_{\alpha s t}\right)$ does not belong to $V\left(f_{0}\right) \cup V\left(f_{p}\right) \cup V\left(\sum_{i=0}^{p} f_{i}\right)$, and belongs to the regular sheet, we see that $\left(\varphi_{z s t}\right)$ is of the form $H \oplus R\left(\Sigma p_{i} \mathbf{e}_{i}\right)$, with $H$ homogeneous, and uniquely determined by its regular composition factors, according to Theorem 3.5. But it is clear that the regular composition factors which are homogeneous, are determined by the value of $f\left(\varphi_{x s t}\right)$. This shows that the restriction of $f$ to the complement of $V\left(f_{0}\right) \cup V\left(f_{p}\right) \cup V\left(\sum_{i=0}^{p} f_{i}\right)$ in $\mathbb{A}[\Gamma, \mathbf{d}]^{0}$ is injective on
the set of $G$-orbits.

Remark. It is easy to derive from Theorem 3.5 the precise number of orbits in any fibre of $f$.

For brevity, let $V_{0}=V\left(f_{0}\right), V_{\infty}=V\left(f_{p}\right), V_{1}=V\left(\sum_{i=0}^{p} f_{i}\right)$. Recall that $n_{t}$ is the number of simple regular representations in $\mathscr{R}_{t}$, and for our given $\mathbf{d}=p \mathbf{h}$ $+\sum_{i \in I} p_{i} \mathbf{e}_{i}$, let $m_{t}$ be the number of different $i \in I$ with $p_{i} \neq 0$ and $E_{i} \in \mathscr{R}_{i}$. Then the number of orbits in $\mathbb{A}[\Gamma, \mathbf{d}]^{0}$ which map to the same point in $V_{0} \backslash\left(V_{1} \cup V_{\infty}\right)$ is just $n_{0}-m_{0}$, similarly, the number of orbits in $\mathbb{A}[\Gamma, \mathbf{d}]^{0}$ which map to a point in $V_{1} \backslash\left(V_{0} \cup V_{\alpha}\right)$ or $V_{x} \backslash\left(V_{0} \cup V_{1}\right)$ is $n_{1}-m_{1}$ or $n_{x}-m_{x}$, respectively.

For $p \geqq 2$, the number of orbits in $\mathbb{A}[\Gamma, \mathbf{d}]^{\circ}$ which map to the same point in $\left(V_{0} \cap V_{1}\right) \backslash V_{\infty}$ is $\left(n_{0}-m_{0}\right)\left(n_{1}-m_{1}\right)$, etc, and for $p \geqq 3$, the number of orbits in $\mathbb{A}[\Gamma, \mathbf{d}]^{0}$ which map to the same point in $V_{0} \cap V_{1} \cap V_{\infty}$ is $\left(n_{0}-m_{0}\right)\left(n_{1}-m_{1}\right)\left(n_{\curvearrowright}\right.$ $\left.-m_{x}\right)$.

\section{References}

1. Auslander, M., Platzek, M.I., Reiten, I.: Coxeter functors without diagrams. Trans. Amer. Math. Soc. 250, 1-46 (1979) 
2. Bernstein, I.N., Gelfand, I.M., Ponomarev, V.A.: Coxeter functors and Gabriel's theorem. Uspechi Mat. Nauk 28, 19-33 (1973), transl. Russ. Math. Surveys 28, 17-32 (1977)

3. Borho, W., Kraft, H.: Über Bahnen und deren Deformationen bei linearen Aktionen reduktiver Gruppen, Comm. Helv. 54, 61-104 (1979)

4. Dlab, V., Ringel, C.M.: Indecomposable representations of graphs and algebras. Memoirs Amer. Math. Soc. 173 (1976)

5. Donovan, P., Freislich, M.R.: The representation theory of finite graphs and associated algebras. Carleton Math. Lecture Notes 5 (1973)

6. Gabriel, P.: Unzerlegbare Darstellungen I. Manuscripta Math. 6, 71-103 (1972)

7. Gabriel, P.: Finite representation type is open. In: Springer Lecture Notes 488, 132-155 (1975)

8. Gabriel, P.: Trends in representation theory. Proceedings Ottawa Conference on Representations of Algebras. To appear (1979)

9. Gelfand, I.M., Ponomarev, V.A.: Problems of linear algebra and classification of quadruples of subspaces in a finite-dimensional vector space. Coll. Math. Soc. Bolyai Tihany (Hungary) 5, 163237 (1970)

10. Kac, V.: Infinite root systems, representations of graphs and invariant theory. Inventiones math. 56, 57-92 (1980)

11. Kronecker, L.: Algebraische Reduktion der Scharen bilinearer Formen. Sitzungsber. Akad. Berlin 763-776 (1890)

12. Nazarova, L.A.: Representations of quadruples. Izv. Akad. Nauk SSSR, Ser. mat. 31, 1361-1377 (1967)

13. Nazarova, L.A.: Representations of quivers of infinite type. Izv. Akad. Nauk SSSR, Ser. mat. 37, 752-791 (1973)

14. Ringel, C.M.: Representations of $k$-species and bimodules. J. Algebra 41, 269-302 (1976)

Received March 7/November 30, 1979 